\title{
A new applicator design for endocavitary brachytherapy of cancer in the nasopharynx
}

\author{
Peter C. Levendag ${ }^{a}, *$, Rob Peters ${ }^{b}$, Cees A. Meeuwis ${ }^{c}$, Leo L. Visch ${ }^{b}$, \\ Dick Sipkema $^{a}$, Connie de Pan ${ }^{\mathrm{a}}$, Paul I.M. Schmitz \\ a Department of Radiation Oncology, Daniel den Hoed Cancer Center/Dijkzigt Hospital, University Hospital Rotterdam, Rotterdam, The Netherlands \\ ${ }^{b}$ Department of Dental Oncology and Maxillofacial Prosthetics, Daniel den Hoed Cancer Center/Dijkzigt Hospital, \\ University Haspital Rotterdam, Rotterdam, The Netherlands \\ 'Department of Head and Neck Surgery, Daniel den Hoed Cancer Center/Dijkzigt Hospital, University Hospital Rotterdam, Rotterdam, The Netherlands \\ ${ }^{d}$ Department of Medical Statistics, Daniel den Hoed Cancer Center/Dijkzigt Hospital, University Hospital Rotterdam. Rotterdam, The Netherlands
}

Received 6 January 1997; revised version received 29 May 1997; accepted 12 June 1997

\begin{abstract}
Introduction: In attempting to improve local tumor control by higher doses of radiation, there has been a resurgence of interest in the implementation of brachytherapy in the management of primary and recurrent cancers of the nasopharynx. Brachytherapy with its steep dose fall-off is of particular interest because of the proximity of critical dose limiting structures. Recent developments in brachytherapy, such as the introduction of pulsed-dose-rate and high-dose-rate computerized afterloaders, have encouraged further evolution of brachytherapy techniques.

Materials and methods: We have designed an inexpensive, re-usable and flexible silicone applicator, tailored to the shape of the soft tissues of the nasopharynx, which can be used with either low-dose-rate brachytherapy or high (pulsed)-dose-rate remote controlled afterloaders.

Results and conclusions: This Rotterdam nasopharynx applicator proved to be easy to introduce, patient friendly and can remain in situ for the duration of the treatment (2-6 days). The design, technique of application and the first consecutive 5 years of clinical experience in using this applicator are presented. (C) 1997 Elsevier Science Ireland Ltd.
\end{abstract}

Keywords: Nasopharynx; Brachytherapy; Endocavitary; Applicator; Low-dose-rate; High-dose-rate

\section{Introduction}

In cancer of the nasopharynx (NP), stage III-IV disease predominates, comprising approximately $75 \%$ of patients. The most important single prognostic factor is the extent of the primary lesion at the time of presentation [11]. Nasopharyngeal cancer (NPC) is known to be highly responsive to RT. Since NPC is also associated with a relatively high incidence of systemic recurrences, some reports advocate the combination of RT and chemotherapy (CHT) for the locally advanced cases [1]. Despite this, a local relapse still represents the most common type of failure, i.e.

\footnotetext{
* Corresponding author. Dariel den Hoed Cancer Center, Groene Hilledijk 301, 3075 EA Rotterdam, The Netherlands.
}

depending on stage, local recurrence rates range from 30 to $60 \%[7,8,13]$. Evidence suggests that the propensity for a local failure relates in particular to the locally applied dose $[2,12,13,16]$. However, the application of high doses of RT is not without considerable potential morbidity. In recent years there has been a resurgence of interest in the implementation of brachytherapy (BT) in the management of primary or recurrent cancers of the NP $[4,14,15,17]$. To boost the NP with high doses of radiation, BT, with its rapid dose fall-off, is of particular interest because of the proximity of critical dose limiting (neural) structures. In an attempt to treat this secluded anatomical midline structure by $\mathrm{BT}$, the ingenuity of clinical researchers is exemplified by the numerous endeavors to improve on the technique [5]. Moreover, recent developments in BT, such as pulsed-dose- 
rate and high-dose-rate computerized afterloaders, using 3D computer planning systems with optimization capabilities, have even further encouraged the evolution of (endocavitary) BT afterloading techniques [9]. The purpose of this report is to describe the design of a new, flexible nasopharyngeal applicator, tailored to the configuration of the soft tissues of the NP, to be used in combination with high-doserate (HDR) remote controlled afterloading systems. The Rotterdam nasopharynx applicator (RNA) was designed to be patient friendly and therefore easy to introduce as an outpatient procedure and to remain in situ for the duration of the treatment ( $2-6$ days).

\section{Materials and methods}

\subsection{NPC treatment protocol}

With the introduction in the Department of Radiation Oncology of a remote controlled HDR afterloader (MicroSelectron HDR, Nucletron-Oldelft), HDR-BT was routinely implemented as a boost after ERT. Since 1991, for primary T1-3 squamous cell carcinoma (SCC) of the NP without parapharyngeal extension, $60 \mathrm{~Gy}$ is applied by ERT using conventional fractionation (2 Gy per fraction/5 days per week) [6]. After a rest period of 1-2 weeks, a booster dose is given to the primary tumor site by BT, using $3 \mathrm{~Gy}$ per fraction, two fractions per day with a $6 \mathrm{~h}$ interval inbetween the fractions for six fractions in total (total dose (TD) $18 \mathrm{~Gy}$, cumulative dose NP $78 \mathrm{~Gy}$ ). In the case of primary cancers with parapharyngeal extension or $\mathrm{T} 4$ tumors, $70 \mathrm{~Gy}$ is applied by ERT, followed by four fractions of $3 \mathrm{~Gy}$ (two fractions per day, $6 \mathrm{~h}$ interval) by HDR-BT (TD 12 Gy, cumulative dose NP 82 Gy). Lymphnodal disease in the neck is treated with a cumulative dose of $70 \mathrm{~Gy}$. In the case of a recurrent cancer, fractionated HDR-BT only is the preferred treatment mode, with cumulative doses tailored to the individual clinical situation.

\subsection{Design of RNA - a stereolithography model study}

Preferably, the introduction of the RNA and the application of the HDR-BT are performed on an outpatient basis. Given the total number of fractions (4-6), for patient convenience the RNA was designed such that the application needs to be performed only once. The applicator was therefore made out of silicone (type 625, Wacker Chemie, Krommenie, The Netherlands) (flexibility) and remains in situ for the duration of the treatment which varies from 2 to 6 days depending on whether a weekend (no RT given) is included in the total treatment duration (Fig. 1). The inner diameter of the silicone tubing (o.d. 15 french, i.d. 9 french) can accommodate standard afterloading catheters (o.d. 6 french). The shape of the silicone applicator (tubing) should ideally closely conform to the nasopharyngeal vault. If so, the radioactive source can be positioned closer to the base of the skull (BOS) as opposed to the soft palate. Computer tomographic scan data (Siemens Somaton Plus) (3 mm slices) were obtained from two NPC patients (Caucasian, Indonesian) with a complete remission after ERT. From these CT slices, a stereolithographic machine (Materialise NV, Heverlee, Belgium) was able to reproduce a solid anatomical model by lighting successive thin layers of a photopolymer with a computer controlled laser beam. In this case, the threshold of the machine was set such that the model represented the anatomy of the soft tissues. The stereolithographic model was then sagitally sliced in a way which made it possible to study the confinements of the air cavities of the nose and the NP proper. Silicone tubing was positioned in the nose and NP; a silicone flange was constructed in order to fixate the curvature of the tubing at the site of the NP proper and/or to push the applicator tubing towards the NP mucosa, i.e. away from the soft palate. Both tubes were interconnected by a small silicone (perforated) bridge (Fig. 1) which abuts proximally to the nasal septum. No difference was observed between the two patients with regard to the shape of the RNA. After decongestion (R/Xylometazoline $\mathrm{HCl} 1 \%$ ) and topical anesthesia, the RNA can be easily introduced into the NP transorally over 4 french (o.d.) guide tubes [10]. Finally, by a small silicone flange (Fig. 1) the RNA remains fixed in a stable position for the treatment period. The silicone RNA can be re-used after cleansing and sterilizing with steam $\left(120^{\circ} \mathrm{C}\right)$.

\subsection{Dose prescription and optimization}

After insertion of standard afterloading catheters with dummy sources into the RNA (Fig. 2), treatment planning is based on orthogonal radiographs. Patient points representing the target (T), i.e. Na, R and BOS, as well as the critical normal tissues (NT), i.e. OC, P, Re, Pa and C, are depicted on lateral and anterior-posterior simulation films according to a well defined departmental protocol (for definition of position patient points see legend to Fig. 2). The obtained dose distribution is optimized such that $\mathrm{Na}$ (and if possible $\mathrm{R})$ receives a dose of $3 \mathrm{~Gy}$ (reference dose) and the NT

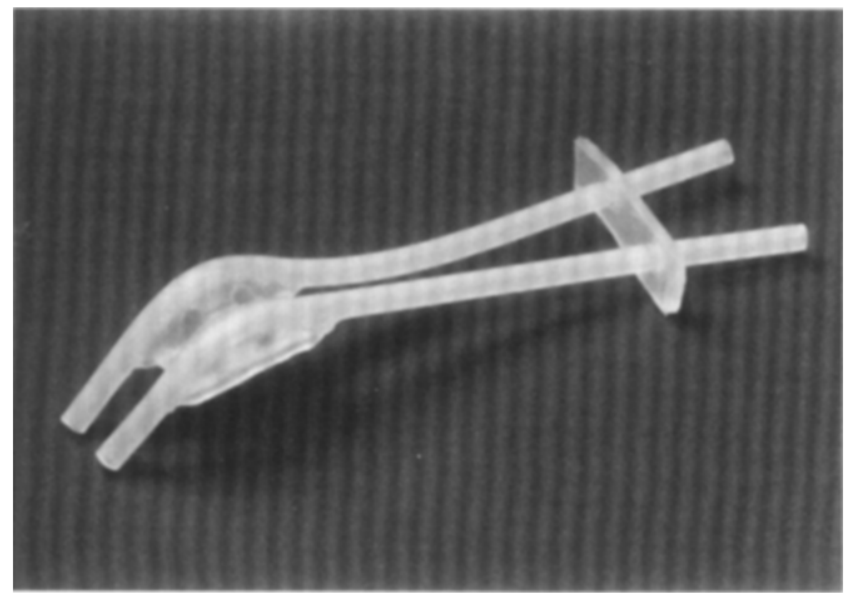

Fig. 1. Standard silicone Rotterdam nasopharynx applicator (RNA). 


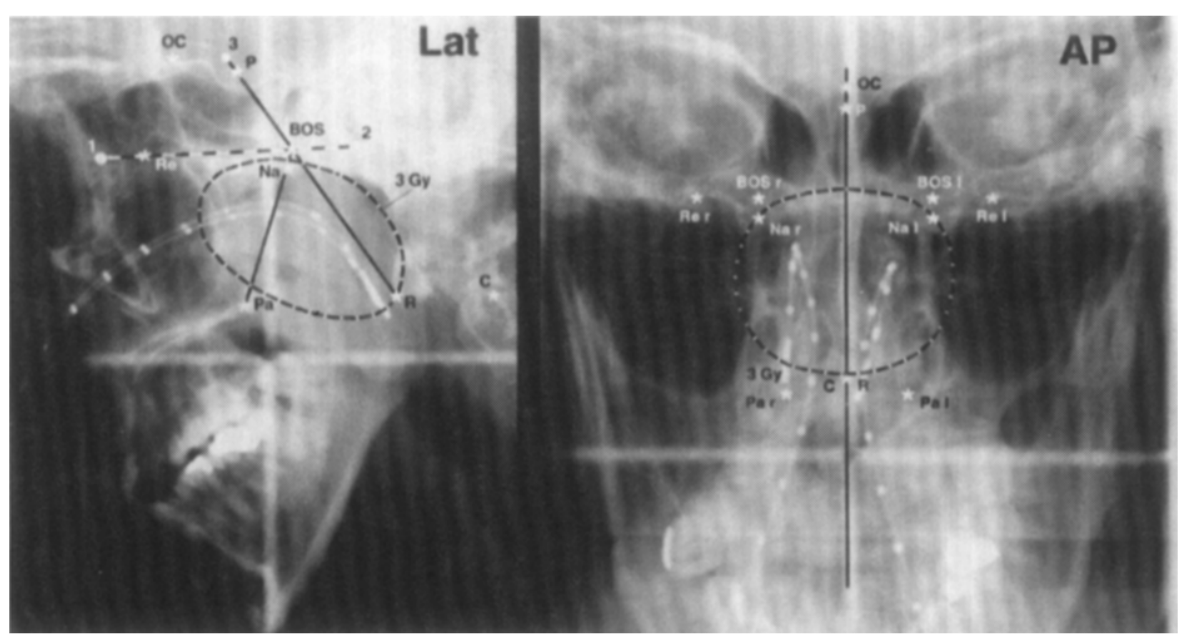

Fig. 2. Lateral (Lat) and anterior-posterior (AP) X-ray simulation films of an NPC patient with RNA (dummy sources inclusive) in situ. After placing lead markers on the (contralateral) outer canthus (1) and tragus (2), a reference line is drawn from (1) to (2);1 cm posterior from (1), a normal tissue point for the retina (Re) is depicted. A second reference line is drawn between the anterior clinoid process ( 3 ) and the position of the node of Rouviere (R) (ventral part corpus C-I). The pituitary gland (P) is positioned $0.5 \mathrm{~cm}$ from (3) (center of sella) on the second reference line; the intersection of line (3-R) with the first reference line (1-2) represents the base of the skull target point (BOS). The optic chiasm (OC) is located on the skull base, $1.5 \mathrm{~cm}$ ventrally from (3). At the junction of the hard and soft palate, a normal tissue point $(\mathrm{Pa})$ is indicated. The intersection of the $\mathrm{Pa}-\mathrm{BOS}$ line with the bony outline of the base of the skull is taken as the target point for prescribing the dose to the nasopharynx (Na). Directly posterior to $\mathrm{R}$, at the posterior border of corpus $\mathrm{C}-\mathrm{I}$, a normal tissue point for the cord $(\mathrm{C})$ is indicated. The $\mathrm{OC}, \mathrm{P}, \mathrm{C}$ and $\mathrm{R}$ points are single midline patient points. $\mathrm{BOS}, \mathrm{Re}, \mathrm{Na}$ and $\mathrm{Pa}$ are bilateral patient points at $1.5,2.5,1.5$ and 1 $\mathrm{cm}$ from the midline, respectively. The stipulated line on the lateral and AP X-ray films represents the 3 Gy (reference) isodose line.

points receive a dose as low as reasonably achievable in terms of the tolerance constraints (Table 1). This is done by assigning different weighting factors to each patient point. After treatment planning, afterloading catheters are introduced into the RNA with each fraction and connected to an HDR afterloading device with a high activity $(370$ GBq) $\mathrm{Ir}^{192}$ stepping point source. From 1991 to 1995,41 consecutive primary NPC patients were treated with ERT and HDR-BT, according to the Rotterdam NPC protocol, testing the efficacy of the RNA applicator. Out of the 41 cases, three patients presented with $\mathrm{T} 1(7 \%)$, nine patients presented with $\mathrm{T} 2(22 \%), 17$ patients presented with $\mathrm{T} 3$ (42\%) and 12 patients presented with T4 (29\%) tumur stages. Thirty-three patients $(81 \%)$ were diagnosed with lymph nodes in the neck.

\section{Results and discussion}

Some literature data are strongly suggestive of the existence of a dose-response relationship in NPC $[2,3,12$, $13,16,17]$. Therefore, in order to escalate doses and at the same time circumvent potential problems of surpassing the tolerance levels of neighboring critical structures, a combination of ERT (primary tumor and bilateral neck) and BT (for boosting the primary site) seems advantageous. As BT for cancers in the head and neck in our center is given in general by fractionated HDR schedules [9], this created the need for an applicator with a novel design, in particular suitable to remain in situ for the duration of the fractionated BT treatment and to be used in conjunction with an HDR afterloader. After testing the RNA for 5 consecutive years, we feel it is safe to conclude that this flexible (silicone) and re-usable applicator is easy to introduce and can remain in the patient for the usual number of treatment days (2-6 days), with only minimal discomfort, i.e. three patients developed synechie of part of the posterior nasal mucosal linings. This can easily be prevented by adequate packing of the nose for approximately 1 week post-BT. Obviously, the RNA could also be loaded with other types of (low activity)

Table 1

Example of brachytherapy dose distribution, non-optimized and optimized, in target and normal tissue patient points for a single patient treated by HDR endocavitary brachytherapy

\begin{tabular}{lll}
\hline Patient points & $\begin{array}{l}\text { Non-optimized dose } \\
\text { (cGy) }(\%)\end{array}$ & $\begin{array}{l}\text { Optimized dose } \\
\text { (cGy) (\%) }\end{array}$ \\
\hline NA (R) & $268(89)$ & $292(97)$ \\
NA (L) & $332(111)$ & $308(103)$ \\
BOS (R) & $146(49)$ & $146(49)$ \\
BOS (L) & $128(43)$ & $128(43)$ \\
R & $366(122)$ & $303(101)$ \\
OC & $45(15)$ & $46(15)$ \\
P & $69(23)$ & $70(23)$ \\
$\operatorname{Re}(R)$ & $48(16)$ & $52(17)$ \\
$\operatorname{Re}(\mathrm{L})$ & $60(20)$ & $61(20)$ \\
Pa (R) & $213(71)$ & $238(79)$ \\
Pa (L) & $253(84)$ & $248(83)$ \\
C & $90(30)$ & $78(26)$ \\
\hline
\end{tabular}

The obtained dose distribution is optimized such that the $\mathrm{Na}$ and $\mathrm{R}$ points receive (approximately) the reference dose of $3 \mathrm{~Gy}$ and the NT points receive a dose as low as can reasonably be achieved. This is done by assigning, by trial and error, different weighting factors for dose requirements to each patient point. L, lcft; R, right. For an explanation of paticnt points, see legend to Fig. 2. 


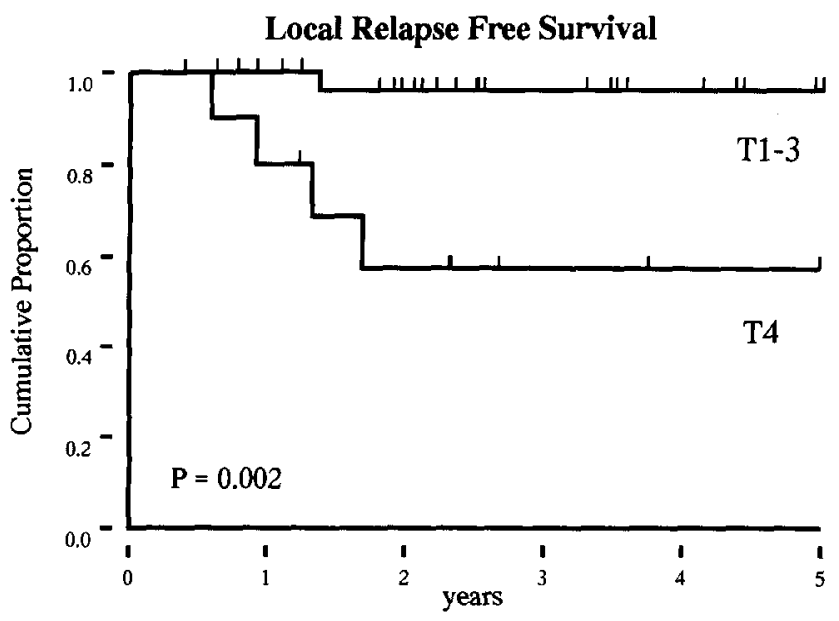

Fig. 3. Local relapse free survival for primary NPC patients treated from 1991 to 1995 in Rotterdam by a combination of ERT and HDR-BT.

radioactive sources, once the outer diameter of the source fits into the standard afterloading catheters.

Since the cumulative doses used were substantial (T3, 78 Gy; T4, 82 Gy), from the start it was felt mandatory to devise a scheme with a number of reproducible patient points, not only in order to optimize the dose to fixed anatomy-based tumor points, but also to try and keep the doses in the critical normal tissues as low as reasonably feasible. For 40 patients we were able to estimate in retrospect (by point dose calculations) the cumulative doses that had been applied by ERT and BT. From the computed median doses (and the dose ranges) for all patient points (data not shown), it was concluded that this type of optimized BT apparently does not lead to overdosing of the critical normal structures.

Finally, in this small consecutive series of 41 patients treated prospectively with a rigidly adhered to HDR-BT protocol, the local relapse free survival (LRFS) of T1-3 $(n=29)$ cancers at 5 years was $96 \%$ versus $57 \%$ for T4 $(n=12)$ tumors $(P=0.002)$ (Fig. 3). Similar findings have been reported by others [3,17]; e.g. for T1-3 tumors treated by ERT combined with low-dose-rate BT at 5 years an LRFS of $91 \%$ was found versus $60 \%$ for those treated by ERT only [17]. Apparently, it seems that BT might play a particularly important role in T1-3 cancers. At present, we are in the process of performing a formal multivariate analysis for all NPC paticnts treated betwcen 1965 and 1995 in our cancer center. In that report we will specifically address the clinical impact of BT.

\section{Acknowledgements}

The authors greatly appreciate the cooperation of Dr R. Berkovits, ENT-surgeon and Drs Jansen, Senan and Eijkenboom, radiation oncologists, in helping to construct and evaluate the Rotterdam nasopharynx applicator. Also, the secretarial work of Ms Inge Dijkstra is much appreciated.

\section{References}

[1] Al-Sarraf, M. and McLaughlin, P.W. Nasopharynx carcinoma: choice of treatment. Int. J. Radiat. Oncol. Biol. Phys. 33: 761-763, 1995.

[2] Bedwinek, J.M., Perez, C.A. and Keys, D.J. Analysis of failures after definitive irradiation for epidermoid carcinoma of the nasopharynx. Cancer 45: 2725-2729, 1990.

[3] Chang, J.T., See, L.C., Tang, S.G.J., Lee, S.P., Wang, C.C. and Hong, J.H. The role of brachytherapy in early-stage nasopharyngeal carcinoma. Int. J. Radiat. Oncol. Biol. Phys. 36: 1019-1024, 1996.

[4] Choy, D., Sham, J.S.T., Wei, W.I., Lo, D., Ho, C.M. and Wu, P.M. Transpalatal insertion of radioactive gold grain for the treatment of persistent and recurrent nasopharyngeal carcinoma. Int. J. Radiat. Oncol. Biol. Phys. 25: 505-512, 1993.

[5] Erickson, B.A. and Wilson, F. Nasopharyngeal brachytherapy. Am. J. Clin. Oncol. 16: 424-443, 1993.

[6] Hermanek, P. and Sobin, L.H. TNM Classification of Malignant Tumours, pp. 1-197. LICC International Union Against Cancer, Springer-Verlag, Berlin, 1987.

[7] International Nasopharyngeal Study Group, VUMCA I trial. Preliminary results of a randomized trial comparing neoadjuvant chemotherapy (cisplatin, epirubicin, bleomycin) plus radiotherapy vs. radiotherapy alone in stage IV $(\geq \mathrm{N} 2, \mathrm{M} 0)$ undifferentiated nasopharyngeal carcinoma: a positive effect on progression-free survival. Int. J. Radiat. Oncol. Biol. Phys. 35: 463-469, 1996.

[8] Lee, A.W.M., Poon, Y.F., Foo, W., Law, S.C.K., Cheung, F.K., Chan, D.K.K., Tung, S.Y., Thaw, M. and Ho, J.H.C. Retrospective analysis of 5037 patients with nasopharyngeal carcinoma treated during 1976-1985: overall survival and patterns of failure. Int. J. Radiat. Oncol. Biol. Phys. 23: 261-270, 1992.

[9] Levendag, P.C., Schmitz, P.I.M., Jansen, P.P., Senan, S., Eijkenboom, W.M.H., Sipkema, D.J., Meeuwis, C.A., Kolkman-Deurloo, I.K.K. and Visser, A.G. Fractionated high dose rate \& pulsed dose rate brachytherapy - first clinical experience in squamous cell carcinoma of the tonsillar fossa and soft palate. Int. J. Radiat. Oncol. Biol. Phys., 1997, in press.

[10] Levendag, P.C., Visser, A.G., Kolkman-Deurloo, I.K.K., Eijkenboom, W.M.H. and Meeuwis, C.A. HDR brachytherapy with special reference to cancer of the nasopharynx. In: Brachytherapy from Radium to Optimization, pp. 121-131. Editors: R.F. Mould, J.J. Battermann, A.A. Martinez and B.L. Speiser. Nucletron International, Veenendaal, 1994.

[11] Liu, T. Trends in the clinical management of nasopharyngeal carcinoma. Int. J. Radiat. Oncol. Biol. Phys. 23: 469-471, 1992.

[12] Mesic, J.B., Fletcher, G.H. and Goepfert, H. Megavoltage irradiation of epithelial tumors of the nasopharynx. Int. J. Radiat. Oncol. Biol. Phys. 7: 447-453, 1981.

[13] Perez, C.A., Devineni, V.R., Marcial-Vega, V., Marks, J.E., Simpson, J.R. and Kucik, N. Carcinoma of the nasopharynx: factors affecting prognosis. Int. J. Radiat. Oncol. Biol. Phys. 23: 271-280, 1992.

[14] Pryzant, R.M., Wendt, C.D., Delclos, L. and Peters, I.J. Re-treatment of nasopharyngeal carcinoma in 53 patients. Int. J. Radiat. Oncol. Biol. Phys. 22: 941-947, 1992.

[15] Teo, P.M.L., Leung, S.F., Choi, P., Lee, W.Y. and Johnson, P.J. Afterloading radiotherapy for local persistence of nasopharyngeal carcinoma. Br. J. Radiol. 67: 181-185, 1994.

[16] Vikram, B., Mishra, U.B., Strong, E.W. and Manolatos, S. Patterns of failure in carcinoma of the nasopharynx: I. Failure at the primary site. Int. J. Radiat. Oncol. Biol. Phys. 11: 1455-1459, 1985.

[17] Wang, C.C. Improved local control of nasopharyngeal carcinoma after intracavitary brachytherapy boost. Am. J. Clin. Oncol. 14: 5$8,1991$. 